nately often came to pass, and from which I did not know how, or could not, defend myself, has caused me much suffering."

Berkeley was a haven, but a problematic one at first because Lawrence, while generous and friendly enough to make a paying place for him, could also be volatile and exploitative. Segrè realized that his one salvation was "to do good physics", which he did, although he took pains to keep himself away from Lawrence's total authority by obtaining a teaching appointment in the physics department. The work on plutonium at Berkeley brought Segrè into collaboration with Glenn Seaborg, whom he calls a man of "unbridled personal ambitions" and an "empire builder". He compares Seaborg unfavourably with "other emperors I have known," including Fermi, who "was emperor because subjects and equals ranked him as such". Segrè declares that after the war he switched to nucleon-nucleon interactions because of Seaborg's control of the radiochemical arena.

Segrè relates few details about his actual work in physics, but does provide arresting observations on his approach to experimentation. As a young physicist in Europe, he worked with Pieter Zeeman and Otto Stern, learning thoroughness from the former and rigour from the latter, but from each "more in the philosophy of experimentation than in technical details". He also absorbed a great deal from the simplicity and directness of Fermi's physics, and not only at the laboratory bench. He takes obvious pleasure in recounting Fermi's remark on a lecture by Oppenheimer (whom Segrè found irritatingly pretentious): "Emilio, I must be getting senile. I went to a learned theoretical seminar and could not understand anything except the last words, which were, 'And this is Fermi's theory of beta decay.",

For many years Segrè pursued what he called "physics without apparatus", imaginatively using simple, almost rudimentary techniques to detect forbidden spectral lines, find new chemical elements and separate nuclear isomers. Once he had switched into nucleon-nucleon interactions, he entered the mode of what he calls "battleship experiments" - experiments such as the search for the antiproton that were obvious to do and could be done effectively if one had a high-energy machine and clean techniques. Of course, the techniques and apparatus were not so simple to devise, and Segrè reports that the experimental protocol required agreement among many people about how the results were to be published and credit allocated. The protocols did not satisfy the physicist Oreste Piccioni, who has claimed that Segrè and others stole his ideas, but Ernest Lawrence rejected Piccioni's complaint at the time and Segrè adds nothing to the issue in his autobiography.

A Mind Always in Motion is rich in further anecdotes and tales about, among other topics, family, physicists, politics, and even young love. It is a valuable memoir and a good read - frank, prickly, crisp and captivating, much like the man.

Daniel J. Kevles is in the Division of the Humanities and Social Sciences, California Institute of Technology, Pasadena, California 91125, USA.

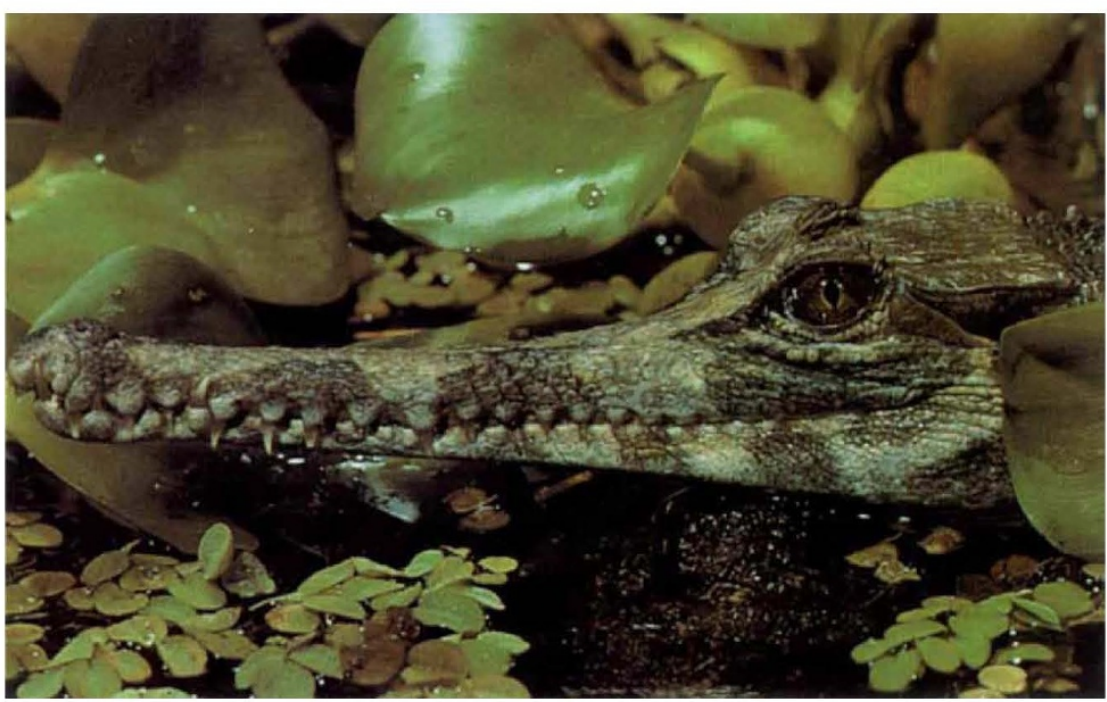

"How cheerfully he seems to grin..." - the rare false gavial is an endangered inhabitant of the Berbak Nature Reserve on the east coast of Sumatra, Indonesia. Although classified with the Crocodylidae family, its long, narrow snout suggests that it is more closely related to the gavial (Gavialis gangeticus) found in parts of southern Asia. From Wetlands in Danger: a World Conservation Atlas edited by Patrick Dugan. Oxford University Press, \$35.

\section{Lake zoobenthos}

\section{Annie Duncan}

A Treatise on Limology: By G. Evelyn Hutchinson, edited by Yvette Edmondson. Wiley: 1993. Pp. 944. £103, \$125.

"To everyone I say farewell"; sadly this is the last book of G. Evelyn Hutchinson and the fourth and last volume of his monumental Treatise on Limnology series. We have waited for this since 1975 when Volume III, Limnological Botany, was published. Meanwhile we have been entertained by his memoirs The Kindly Fruits of the Earth (Yale University Press, 1979) and instructed by his text An Introduction to Population Ecology (Yale University Press, 1978).

The fourth volume of the Treatise on Limnology adds the description of deep lake zoobenthos to the grand scheme of the series, which deals with physicochemistry, planktonic animals and plants and littoral benthos. Hutchinson had plans for a fifth volume but "it soon became clear that a properly comprehensive version of the Treatise would take far more time than is normally allotted to a human life". So, as his editor Yvette Edmondson comments, "it is sad to see what we have missed". The fourth volume was not completed before Hutchinson died but he was wise in his choice of an editor in 1988, and its completion has been a work of love, with Yvette Edmondson being supported in her task by his many students, colleagues and friends.

There are six chapters. The first defines types of zoobenthos which are then treated separately: the attached and unattached haptobenthos (protists, sponges, rotifers and so on), with some consideration on the differing nature of attachment surfaces; then the freely moving gastropod molluscs, followed by the aquatic insects, dealing first with juvenile stages of mayflies, Odonata and stoneflies and then with the bugs and beetles with wholly aquatic adults.

As in the previous volumes, the text is comprehensive, culling and ordering facts from a bibliography of 1,735 titles. Although these titles span the period from 1618 to 1991 , over half were published between 1950 and 1980, when Hutchinson must have been reading more than one scientific paper a day. At the end of every chapter, there is a very useful summary of the main conclusions. The whole series is a grand monument to the scientific life of one man with a "sparkling mind" whose influence will continue after life even more than during it.

Annie Duncan is in the Department of Biology, Royal Holloway, University of London, Egham, Surrey TW2O OEX, UK. 